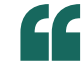

expression of this peptide is regulated by nutrients, with glucose acting as a catabolite repressor

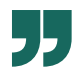

symbiont and the host immune system," explains Allen.

The B $\theta O M$ T cells were found to recognize a single peptide expressed by $B$. theta in response to glycan cues. The researchers then showed that expression of this peptide is regulated by nutrients, with glucose acting as a catabolite repressor. "We found that mice fed a high-glucose diet decreased activation of the symbiontspecific $T$ cells by downregulating the expression of a dominant antigen," reports Allen. "This finding demonstrates that diet can alter $\mathrm{T}$ cell-driven immune responses to microbial antigens."

"We plan to test if our findings can be extended to key antigens in other microorganisms, especially those that are enriched in patients with IBD," concludes Allen. "If dietary regulation of dominant microbial antigens is widespread, such dietary manipulations might become effective therapies for diseases such as IBD."

Iain Dickson

ORIGINAL ARTICLE Wegorzewska, M. M. et al. Diet modulates colonic T cell responses by regulating the expression of a Bacteroides thetaiotaomicron antigen. Sci. Immunol. 4, eaau9079 (2019)

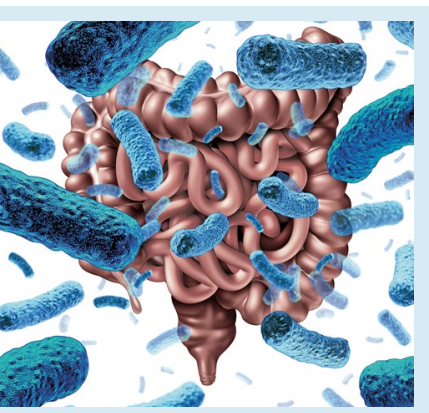

Credit: Brain light / Alamy Stock Photo

increased
abundance
of bacteria
producing
the SCFA
butyrate is
causally linked
to improved
insulin
response

ORIGINAL ARTICLE Sanna, S. et al. Causal relationships among the gut microbiome, short-chain fatty acids and metabolic diseases. Nat. Genet. https://doi.org/10.1038/ s41588-019-0350-x (2019)

\section{STEM CELLS}

\section{IL-22 assures gut homeostasis}

A new report in Nature shows that IL-22 produced by tissueresident innate lymphocytes in response to environmental stimuli protects intestinal stem cells (ISCs) against genotoxic stress and mutations through regulation of their DNA damage response (DDR) machinery.

To prevent malignant transformation and tumour development following exposure to genotoxins, ISCs maintain integrity of their genome via the DDR, which results in DNA repair or apoptosis. Previous studies had indicated a role for IL-22 in intestinal carcinogenesis, albeit with conflicting findings. A new paper by Konrad Gronke and colleagues set out to elucidate the pathways and effects of IL-22 signalling on carcinogenesis via stem cells in the intestinal crypt using a new mouse model. "In most studies two mouse strains are compared (for example, wild-type versus genedeficient) but these mice might differ in their microbiota or inflammatory tonus, affecting data interpretation," explains Andreas Diefenbach, senior author of the study. "We generated mice with mosaic expression of the IL-22 receptor within the intestinal epithelium, resulting in mice in which some crypts lost IL-22 receptor expression next to crypts that retained it. This approach enabled analysis of IL-22 effects on the epithelium within one mouse, mitigating any confounding effects."

When mice were treated with a carcinogen followed by dextran sodium sulfate to induce tumorigenesis, those without the IL-22 receptor in some crypts had an increased fraction of tumours, suggesting that absence of IL-22 signalling predisposes epithelial cells to carcinogenesis. Transcriptional profiling showed that ISCs lacking IL-22 signalling had reduced expression of genes involved in DNA repair, DDR and apoptosis. Investigation of the steps of DDR in

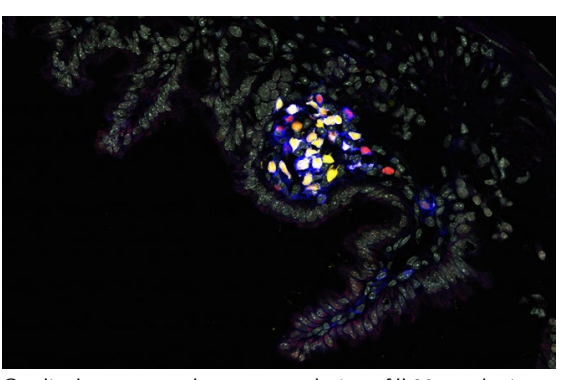

Credit: A cryptopatch, an accumulation of IL22-producing ILC3s, in the small intestine of mice (ILC3s labelled by a reporter allele (yellow), and by staining for ROR $\gamma \mathrm{t}$ (red) and CD4 (blue)). Image courtesy of K. Gronke, Charité-Universitätsmedizin Berlin, Germany.

ISCs following exposure to genotoxic stress demonstrated that IL-22 has a role in DDR initiation via induction of ATM expression through STAT3 signalling. Further work suggested that increased tumorigenesis in the absence of IL-22 is caused by a decreased rate of apoptosis and by accumulation of mutations.

In the colon, $\mathrm{CD}^{+} \mathrm{T}$ cells and group 3 innate lymphoid cells (ILC3s) are the main producers of IL-22, followed by FOXP ${ }^{+} \mathrm{CD} 4{ }^{+} \mathrm{T}$ cells and $\gamma \delta$ T cells. Glucosinolates are phytochemicals found in cruciferous vegetables, of which some are potent genotoxins. The researchers found that treatment of mice with a glucosinolate metabolite increased IL-22 production by ILC 3 s and $\gamma \delta$ T cells, which was dependent on aryl hydrocarbon receptor signalling. When mice with intact IL-22 signalling were fed a diet without glucosinolates, their colonic ILC 3 s and $\gamma \delta$ T cells did not produce IL-22 and epithelial cells had an impaired DDR following DNA damage.

"Our study adds to findings that the roles of the immune system go beyond defense against pathogens. The data reveal a homeostatic network by which the immune system senses genotoxic compounds contained in nutrients and generates an output (IL-22) that adapts stem cell function to cope with genotoxic stress," summarizes Diefenbach. "The findings might stimulate further research into how dietary compounds might influence the intestinal immune status."

Clemens Thoma 